\title{
Ventricular Run by ECG Finding
}

National Cancer Institute

\section{Source}

National Cancer Institute. Ventricular Run by ECG Finding. NCI Thesaurus. Code C120621.

An electrocardiographic tracing demonstrating three or more consecutive QRS

complexes of ventricular origin, irrespective of rate. 